\title{
Prophylactic Role of Curcumin against Tubular Atrophy caused by Aspirin in Kidneys of Adult Female Albino Rats
}

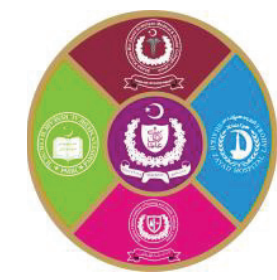

${ }^{1}$ Saba Amjad, ${ }^{2}$ Muhammad Suhail, ${ }^{3}$ Saima Malik, ${ }^{2}$ Tayyaba Muzaffar, ${ }^{2}$ Javaid Iqbal, ${ }^{2}$ Hadia Zulfiqar

${ }^{1}$ Department of Anatomy, UCMD, University of Lahore

${ }^{2}$ Department of Anatomy, Federal Postgraduate Medical Institute, Lahore

${ }^{3}$ Department of Anatomy, University Medical \& Dental College, Faisalabad

\begin{abstract}
Introduction: Aspirin the inhibitor of prostaglandin and thromboxane synthesis is used to relieve fever, inflammation and pain. Curcumin that exhibits anti-inflammatory, antioxidant and anti-atherosclerotic properties is obtained from the rhizome of curcuma longa plant. Aims \& Objectives: To evaluate the nephroprotective effects of curcumin against tubular atrophy caused by aspirin in kidneys of adult female albino rats. Place and duration of study: The study was conducted in Anatomy Department of Federal Postgraduate Medical Institute, Lahore for 30 days. Material \& Methods: 4 groups were made containing 15 rats in each group. Nephrotoxicity in the rats was induced with aspirin given by orogastric intubation (200 $\mathrm{mg} / \mathrm{kg}$ bodyweight) in positive control group B and experimental groups C \& D. Then rats of experimental groups C\& D in addition to aspirin received $15 \mathrm{mg} / \mathrm{kg} \mathrm{\& 30} \mathrm{mg/kg} \mathrm{body} \mathrm{weight} \mathrm{of} \mathrm{curcumin} \mathrm{by} \mathrm{orogastric}$ intubation. Results: On histopathological examination, the proximal and distal convoluted tubules were observed normal in control group A (Fig-1) Atrophic changes in the proximal and distal convoluted tubules along with damage to the tubular epithelial cells were found in 11 rats $(73.3 \%)$ in positive control group B, 4 rats $(26.7 \%)$ in experimental group $\mathrm{C}$ and 3 rats $(20.0 \%)$ in experimental group D (Fig-2, $3 \& 4$ ). The difference was found highly significant with p-value $<0.001$ (Table-1). Conclusion: The present study has shown that curcumin exerts profound effects in reducing the damage to proximal and distal convoluted tubules caused by aspirin in a dose dependent manner.
\end{abstract}

Key words: Aspirin, curcumin, tubular atrophy, proximal convoluted tubules, distal convoluted tubules, kidneys, adult female albino rats

\section{INTRODUCTION}

Aspirin has emerged as a cornerstone therapy available for treating pain, inflammation and cardiovascular diseases. Its history dates back to the use of willow bark by the Egyptian healers. Aspirin produces nephrotoxicity by inhibiting the synthesis of prostaglandins. ${ }^{1}$ It inhibits the activity of the enzyme cyclooxygenase-1 (COX-1) and cyclooxygenase-2 (COX-2). ${ }^{2}$ Aspirin causes atrophy of tubular epithelial cells by ischemia, atrophic changes in the proximal \& distal convoluted tubules in the renal cortex and the inflammatory changes in the renal medulla. ${ }^{3}$

Medullary ischemia is the initial event in the development of nephrotoxicity. NSAIDs inhibit prostaglandin synthesis that decreases renal medullary blood flow. ${ }^{4}$ Studies have shown that sexually mature female rats are more predisposed to aspirin induced nephrotoxicity than male and immature female rats. ${ }^{5}$ It has been found that females have lower concentration of serum aspirin esterase levels which degrade aspirin more slowly. ${ }^{6}$ Aspirin has various therapeutic uses in the prevention of cardiovascular diseases, the prevention \& treatment of Alzheimer's disease and chemoprevention of colorectal cancer. ${ }^{7}$ It causes gastrointestinal disturbances which include nausea, vomiting, heartburn, indigestion, peptic ulcers and gastrointestinal bleed. Aspirin is contraindicated in patients with peptic ulcer disease, gastrointestinal bleeding, alcoholic intoxication and cerebral hemorrhage. ${ }^{8}$

Turmeric that possesses versatile properties belongs to Zingiberaceae family. ${ }^{9}$ Curcumin a chemical constituent of turmeric powder is a polyphenol obtained from the rhizome of the plant Curcuma Longa. ${ }^{10}$ It is used as a cooking spice and adds 
flavor to the food. ${ }^{11}$ Curcumin is frequently used in traditional Ayurvedic medicine. It has been extensively cultivated in Asian countries predominantly in India and China. ${ }^{12}$ The commercially produced curcuminoid complex is composed of $71.5 \%$ curcumin I (diferuloylmethane), $19.4 \%$ curcumin II (demethoxycurcumin) and $9.1 \%$ curcumin III (bisdemethoxycurcumin). ${ }^{13,14}$ It also contains volatile oils, sugars, proteins and resins. The curcuminoid complex due to its resemblancewith the saffron is also known as "indian saffron" and can be used as a substitute. ${ }^{15}$ Curcumin posseses anti-inflammatory, antiatherosclerotic, ${ }^{16}$ antibacterial, antioxidant, antispasmodic, anti-protozoal, antihuman immunodeficiency virus and nematocidal activities. ${ }^{12}$ The beneficial effects of curcumin were documented in one study for the treatment of swelling and sprains when applied locally over the affected area. ${ }^{17}$ Curcumin helps in wound healing and muscle regeneration, prevents gall stone formation and cataract, inhibits scarring, prevents liver and kidney toxicity, septic shock, cardiovascular disease, inflammatory bowel disease, ${ }^{18}$ and arthritis. ${ }^{19}$ Curcumin restores brain derived neurotropic factors thus causing significant reversal of depression like behavior. ${ }^{18}$ It prevents DNA damage by inhibiting free radical generation and lipid peroxidation. ${ }^{20}$ Curcumin reduces the profibrotic cytokines, VEGF, transforming growth factor-beta (TGF- $\beta$ ), connective tissue growth factor (CTGF), extracellular matrix proteins, fibronectin and collagen type IV. It also prevents structural damage by reducing glomerulosclerosis Index (GI), tubulointerstitial fibrosis and arteriolopathy. ${ }^{21}$ Curcumin down regulates the activity of cytochrome P450 that can lead to toxicity of some drugs. ${ }^{22}$ Various evidences indicate that curcumin acts as a bifunctional antioxidant with both direct and indirect antioxidant effects, such as scavenging reactive oxygen species and inducing the expression of cytoprotective proteins. Hence preventing oxidative stress and reducing anti-oxidant enzymes ${ }^{23}$ which could be beneficial against drug induced nephrotoxicity. In previous studies the prophylactic role of curcumin against other nephrotoxic drugs has been observed.

The present study was designed to evaluate the prophylactic effects of curcumin against tubular atrophy caused by aspirin in kidneys of adult female albino rats.

\section{MATERIAL AND METHODS}

This experimental study was conducted in the Department of Anatomy, Federal Postgraduate Medical Institute, Lahore.

All rats were kept in cages in the animal house of the Department of Anatomy, PGMI, Lahore. The rats were allowed free access to food and water. A 12 hourly light: dark cycle was maintained at room temperature of $27^{\circ} \mathrm{C}$. After acclimatization for 15 days, these rats were kept in separate cages divided into 4 groups A, B, C and D with proper labeling. 15 rats were distributed in each group. Body weight was measured before and at the end of experiment. Blood samples were taken from the rat's tail before the experiment and then on $15^{\text {th }}$ and $30^{\text {th }}$ day of experiment, for serum creatinine levels of all four groups. A lignocaine cream as a local anaesthetic was applied to the site 30 minutes prior to blood sampling. ${ }^{24}$

Turmeric extract was prepared from PCSIR laboratory, Lahore. Quantification of extract was done through Gas Chromatography-Mass Spectrometry (GC-MS) from Chemistry Department of Forman Christian College, Lahore.

Turmeric extract was given to the rats through orogastric intubation.

Group A (Healthy Control Group): The rats received $10 \mathrm{ml} / \mathrm{kg}$ body weight/day of distilled water.

Group B (Positive Control Group): 100mg/kg body weight/day of aspirin for 30 days was given.

Group C (Experimental Group I): $100 \mathrm{mg} / \mathrm{kg}$ body weight/day of aspirin and $15 \mathrm{mg} / \mathrm{kg}$ body weight/day of curcumin simultaneously for 30 days were given.

Group D (Experimental Group II): $100 \mathrm{mg} / \mathrm{kg}$ body weight/day of aspirin and $30 \mathrm{mg} / \mathrm{kg}$ body weight/day of curcumin simultaneously for 30 days were given.

After dissection, the kidneys were removed and studied histologically for the tubular damage.

\section{Statistical analysis:}

The data was entered and analyzed by using SPSS 22.0. The qualitative data for the tubular damage was stated by using frequency \& percentage of each group. Chi-square test was used for the comparison among groups. P-value $\leq 0.05$ was considered statistically significant. 


\section{RESULTS}

The proximal and distal convoluted tubules were found normal in control group A (Fig-1) Atrophic changes in the proximal and distal convoluted tubules along with damage to the tubular cells were observed in 11 rats $(73.3 \%)$ in positive control group $\mathrm{B}$ followed by 4 rats $(26.7 \%)$ and 3 rats $(20.0 \%)$ in experimental groups C \& D respectively (Fig-2, $3 \& 4$ ). The difference was found highly significant with p-value $<0.001$. (Table- 1 )

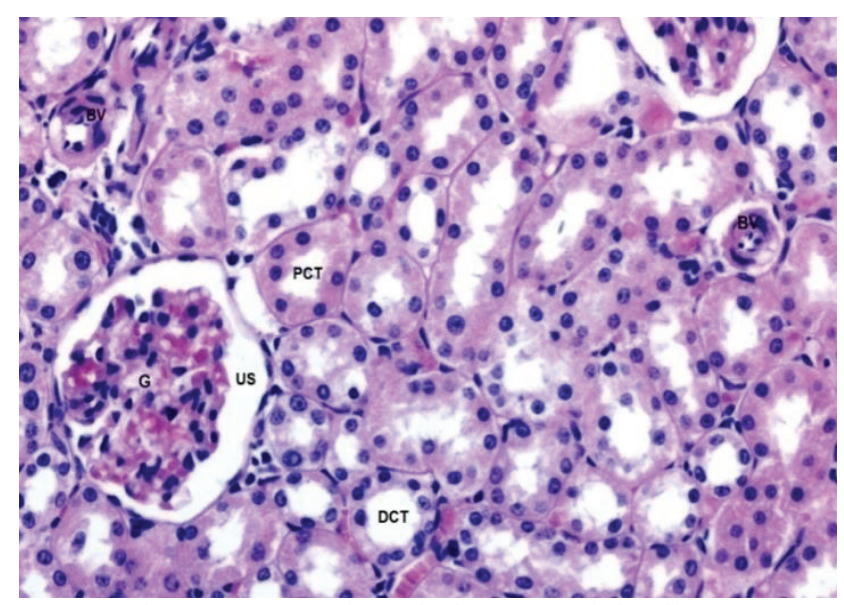

Fig-1: Photomicrograph of kidney of adult albino rat of control group A showing: Glomerulus(G), Urinary space (US), Blood vessel (BV), Proximal convoluted tubule (PCT) and Distal convoluted tubule (DCT) (H\&E,20x)

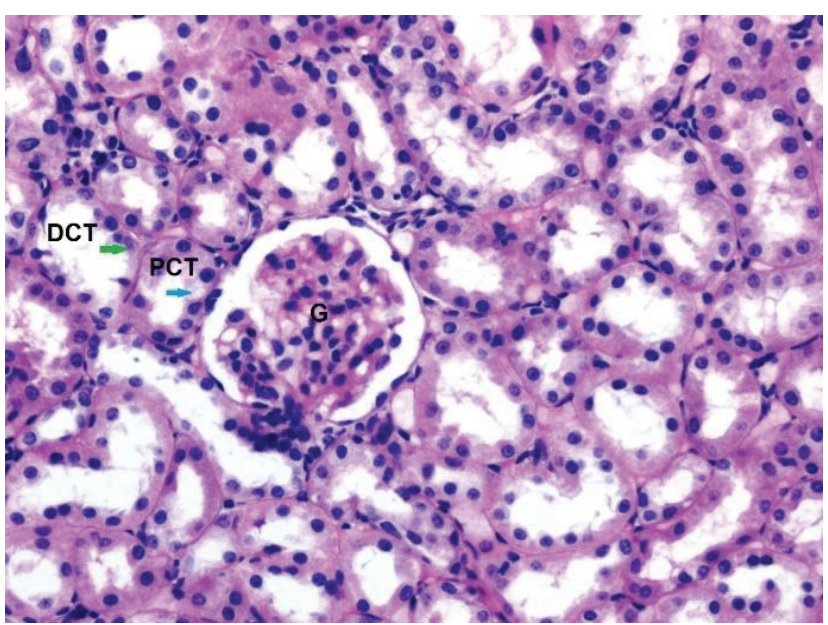

Fig-2: Photomicrograph of kidney of adult albino rat of positive control group B showing: Glomerulus $(\mathrm{G})$, cytoplasmic vacuolization of Proximal convoluted tubule (blue arrow) and tubular atrophy of Distal convoluted tubule (green arrow). (H\&E,20x)

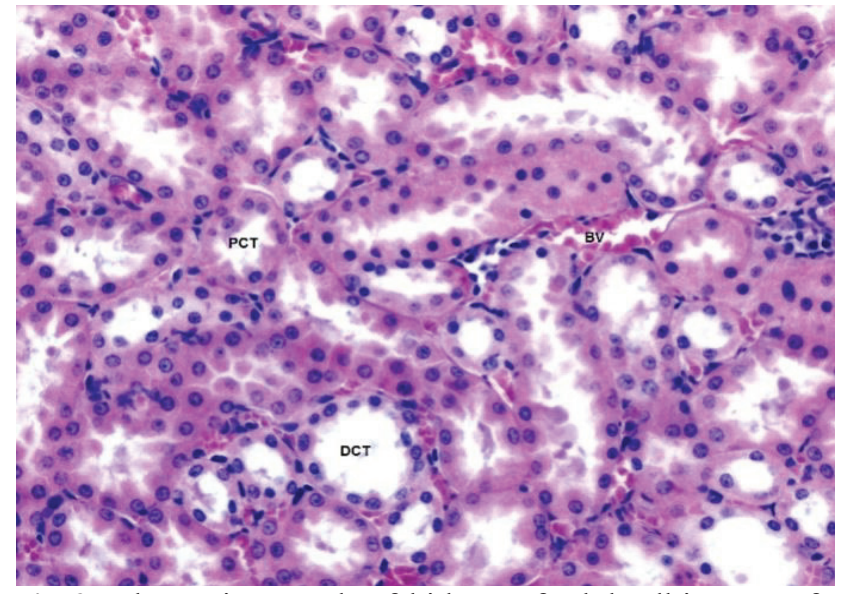

Fig-3: Photomicrograph of kidney of adult albino rat of experimental group $\mathrm{C}$ showing less changes in: Blood Vessels (BV), Proximal convoluted tubule \& Distal convoluted tubule. (H\&E,20x)

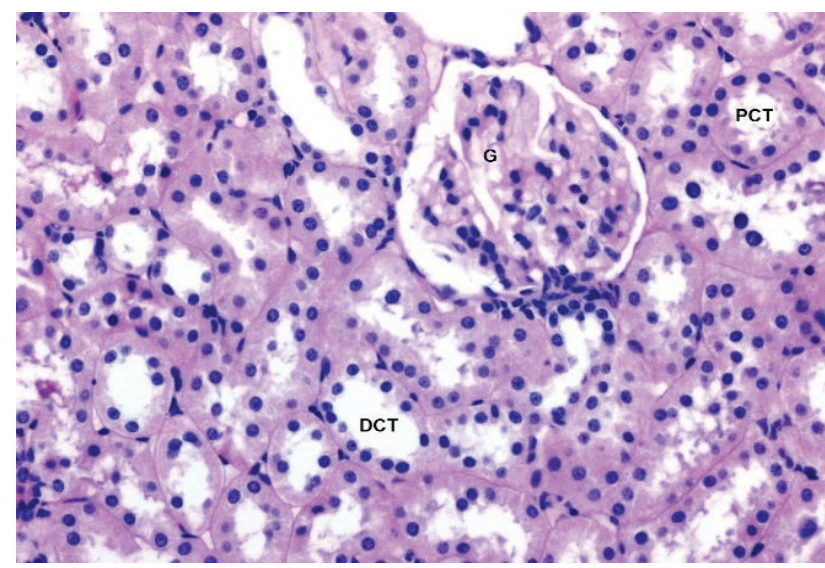

Fig-4: Photomicrograph of kidney of adult albino rat of experimental group D showing: Glomerulus (G), leastchanges in the Proximal convoluted tubule \& Distal convoluted tubule (H\&E,20x)

\begin{tabular}{|c|c|c|c|c|c|c|}
\hline \multirow{2}{*}{} & \multicolumn{6}{|c|}{ Tubular Damage } \\
\cline { 2 - 7 } & \multicolumn{2}{|c|}{ Present } & \multicolumn{2}{c|}{ Absent } & \multicolumn{2}{c|}{ Total } \\
\cline { 2 - 7 } & $\mathbf{N}$ & $\mathbf{\%}$ & $\mathbf{N}$ & $\mathbf{\%}$ & $\mathbf{N}$ & $\%$ \\
\hline Group A & 0 & 0.0 & 15 & 100.0 & 15 & 100.0 \\
\hline Group B & 11 & 73.3 & 4 & 26.7 & 15 & 100.0 \\
\hline Group C & 4 & 26.7 & 11 & 73.3 & 15 & 100.0 \\
\hline Group D & 3 & 20.0 & 12 & 80.0 & 15 & 100.0 \\
\hline
\end{tabular}

Chi-square $=23.50 \quad$ p-value $<0.001$

Table-1: Comparison of tubular damage among control groups given aspirin and experimental groups given aspirin \& curcumin

The group wise comparison of tubular damage revealed a significant difference among control group $\mathrm{A} \&$ experimental groups $\mathrm{C}$ and $\mathrm{D}$ from positive control group B with p-values $<0.001$, 0.028 and 0.010 respectively. The difference between control group A and experimental group C had a p-value 0.100. (Table-2 \& Fig-5) 


\begin{tabular}{|c|c|c|c|c|}
\hline (I) Group & (J) Group & Chi-square & Df & P-value \\
\hline \multirow{3}{*}{ Group A } & Group B & 14.35 & 1 & $<0.001^{* *}$ \\
\cline { 2 - 5 } & Group C & 2.60 & 1 & 0.100 \\
\cline { 2 - 5 } & Group D & 1.48 & 1 & 0.224 \\
\hline \multirow{2}{*}{ Group B } & Group C & 4.80 & 1 & $0.028^{*}$ \\
\cline { 2 - 5 } & Group D & 6.56 & 1 & $0.010^{*}$ \\
\hline Group C & Group D & 0.00 & 1 & 1.000 \\
\hline
\end{tabular}

Table-2: Group wise comparison of tubular damage among control groups given aspirin and experimental groups given aspirin \& curcumin (by using chi-square)

KEY

A Control Group of rats

B Positive control Group of rats

C Experimental Group I of rats

D Experimental Group II of rats

$\mathrm{N}$ Number of rats in each group

Df Degrees of freedom

** Highly significant difference $(\mathrm{P}<0.001)$

* Significant difference $(\mathrm{P}<0.05)$

$+\quad$ Insignificant difference $(\mathrm{P}>0.05)$

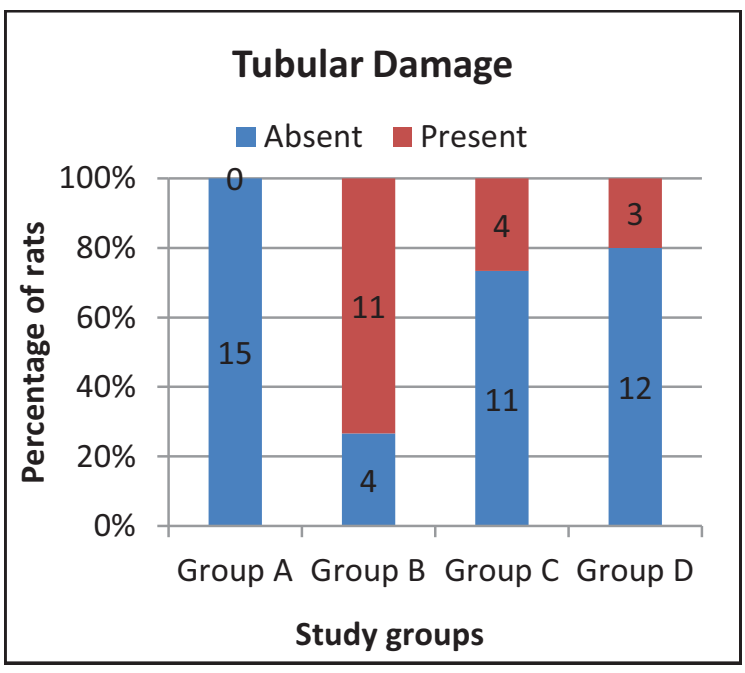

Fig-5: Graphic comparison of tubular damage among control groups given aspirin and experimental groups given aspirin $\&$ curcumin

\section{DISCUSSION}

Aspirin has been used as a famous, cheap and readily available drug. It shows versatile properties such as an anti-inflammatory, anti-platelet, antipyretic \& analgesic actions. ${ }^{3,8}$ Curcumin is a spice, additive and a pigment commonly used in Asia. ${ }^{25}$

In the present study the atrophic changes in the proximal and distal convoluted tubules along with damaged tubular epithelial cells in the cortex of the kidney were found in more number of rats in positive control group B followed by experimental groups $\mathrm{C}$ and $\mathrm{D}$. The difference of damage of proximal and distal convoluted tubules among the four groups was found significant with $\mathrm{p}$-value
$<0.001$ (Table-1). These observations were consistent with the results of the study performed by Molland et al. who observed tubular atrophy and an interstitial inflammatory cell infiltrate after aspirin administration. It may be secondary to tubular obstruction by fibrosis or may involve a direct toxic effect of aspirin on cortical tubules. ${ }^{26}$ These results are also similar with the findings performed by Neha et al, Owen et al \& Calder et al who proved that aspirin causes damage of the proximal and distal convoluted tubules in the cortex.. ${ }^{27}$ The underlying mechanism of tubular damage involves tubular vacuolization. It arises due to hypoxia, mitochondrial injury, free radical production and decreased production of ATP. ${ }^{28}$ Acute tubular injury involves attenuation of the brush borders of the proximal tubules and detachment of the tubular cells from the underlying basement membranes. ${ }^{29}$ TGFbeta has been shown to play a major role in the induction of tubular atrophy. ${ }^{30}$

Less tubular changes were observed in the experimental groups C \& D given curcumin in addition to aspirin. These findings are in relevance with the results of Tapia et al who states that curcumin has regenerative potential due to its antioxidant properties. ${ }^{31}$ Administration of curcumin restores the degenerated renal tubules and preserve normal renal structure. ${ }^{32}$

\section{CONCLUSION}

The results of the present study indicated that the co-treatment of aspirin with the ethanolic extract of turmeric prevented aspirin induced damage of the proximal and distal convoluted tubules in adult female albino rats.

\section{REFERENCES}

1. Fuster V, Sweeny JM. Aspirin a historical and contemporary therapeutic overview. Circulation. 2011; 123(7):768-78.

2. Aspirin 2014 [updated 2014 april 07]. Available from: en.wikipedia.org/wiki/Aspirin

3. Jain Neha, Shrivastava Renu, Raghuwanshi Arun K and Shrivastava Vinoy K. Aspirin induced changes in serum ACP, ALP, GOT, GPT, Bilirubin and Creatinine in correlation with histopathological changes in liver and kidney of female albino rat. Int J App Pharm. 2012; (4):9-11

4. Ejaz P, Bhojani K, Joshi V. NSAIDs and kidney. JAPI. 2004; 52:632-9.

5. Nanra RS, Kincaid-Smith P. Papillary necrosis in rats caused by aspirin and aspirin-containing 
mixtures. British medical journal. 1970; 3(5722):559.

6. Menguy R, Desbaillets L, Masters YF, Okabe S. Evidence for a sex-linked difference in aspirin metabolism. 1972.

7. Vainio H, Morgan G. Aspirin for the second hundred years: new uses for an old drug. Pharmacology \& toxicology. 1997; 81(4):151-2.

8. Awtry EH, Loscalzo J. Aspirin. Circulation. 2000; 101(10):1206-18.

9. Shimatsu A, Kakeya H, Imaizumi A, Morimoto T, Kanai M, Maeda S. Clinical application of "curcumin", a multi-functional substance. AntiAging Med. 2012; 9:43-51.

10. Aggarval B, Kumar A, Bharti A. Anticancer potential of curcumin: preclinical and clinical studies. Anticancer Res. 2003; 23:363-398.

11. Li S, Yuan W, Deng G, Wang P, Yang P, Aggarwal BB. Chemical composition and product quality control of turmeric (Curcuma longa L.). Pharmaceutical Crops. 2011; 2:28-54.

12. Araujo C, Leon L. Biological activities of Curcuma longa L. Memórias do Instituto Oswaldo Cruz. 2001; 96:723-8.

13. Li S, Yuan W, Deng G, Wang P, Yang P, Aggarwal BB. Chemical composition and product quality control of turmeric (Curcuma longa L.). Pharmaceutical Crops. 2011; 2:28-54.

14. Chattopadhyay I, Biswas K, Bandyopadhyay U, Banerjee RK. Turmeric and curcumin: Biological actions and medicinal applications. Current science. 2004; 87:44-53.

15. Julie S, Jurenka M. Anti-inflammatory Properties of Curcumin, a Major Constituent. Alternative Medicine Review. 2009;14:141-153.

16. Aggarwal BB, Harikumar KB. Potential therapeutic effects of curcumin, the antiinflammatory agent, against neurodegenerative, cardiovascular, pulmonary, metabolic, autoimmune and neoplastic diseases. The international journal of biochemistry \& cell biology. 2009; 41:40-59.

17. Ammon H, Wahl M. Pharmacology of Curcuma longa. Planta medica. 1991; 57(1):1.

18. Beevers CS, Huang S. Pharmacological and clinical properties of curcumin. Botanics: Targets Ther. 2011; 1:5-18.

19. Khanna D, Sethi G, Ahn KS, Pandey MK, Kunnumakkara AB, Sung B, et al. Natural products as a gold mine for arthritis treatment. Current Opinion in Pharmacology.2007;7:344-51

20. Sikora E, Bielak-Zmijewska A, Piwocka K, Janusz S, Radziszewska E. Inhibition of proliferation and apoptosis of human and rat $\mathrm{T}$ lymphocytes by curcumin, a curry pigment. Biochemical pharmacology. 1997; 54:899-907.

21. Epstein J, Sanderson IR, MacDonald TT. Curcumin as a therapeutic agent: the evidence from in vitro, animal and human studies. British journal of nutrition. 2010; 103:1545-57.

22. Burgos-Morón E, Calderón-Montaño JM, Salvador J, Robles A, López-Lázaro M. The dark side of curcumin. International Journal of Cancer. 2010; 126:1771-5.

23. Tapia E, Soto V, Ortiz-Vega KM, ZarcoMárquez G, Molina-Jijón E, Cristóbal-García $M$, et al. Curcumin induces Nrf2 nuclear translocation and prevents glomerular hypertension, hyper filtration, oxidant stress, and the decrease in antioxidant enzymes in 5/6 nephrectomized rats. Oxidative medicine and cellular longevity. 2012; 2012.

24. Flecknell P, Liles J, Williamson H. The use of lignocaine-prilocaine local anaesthetic cream for pain-free venepuncture in laboratory animals. Laboratory Animals. 1990; 24:142-6.

25. Coca SG, Singanamala S, Parikh CR. Chronic kidney disease after acute kidney injury: a systematic review and meta-analysis. Kidney international. 2012; 81(5): 442-8.

26. Molland EA. Aspirin damage in the rat kidney in the intact animal and after unilateral nephrectomy. The Journal of pathology. 1976; 120 (1):43-8.

27. Calder I, Funder C, Green C, Ham KN, Tange J. Comparative nephrotoxicity of aspirin and phenacetin derivatives. $\mathrm{Br}$ Med J. 1971; 4 (5786): 518-21.

28. Frazier K, Paredes A, Dube P, Styer E. Connective tissue growth factor expression in the rat remnant kidney model and association with tubular epithelial cells undergoing transdifferentiation. Veterinary pathology. 2000; 37 (4):328-35.

29. Stricker TP, Kumar V, Maitra A, Schoen FJ, Mitchell RN, Aster JC. Robbins Basic Pathology. Neoplasia. 2007; 173:52.

30. Vahed SZ, Samadi N, Ardalan M. Diagnosis of interstitial fibrosis and tubular atrophy in kidney allograft: implementation of microRNAs. Iranian journal of kidney diseases. 2014; 8 (1):4.

31. Tapia E, Zatarain-Barrón ZL, Hernández-Pando R, Zarco-Márquez G, Molina-Jijón E, CristóbalGarcía M, et al. Curcumin reverses glomerular hemodynamic alterations and oxidant stress in 5/6 nephrectomized rats. Phytomedicine. 2013; 20(3): 359-66.

32. Soliman MM, Baiomy AA, Yassin MH. Molecular and histopathological study on the 
ameliorative effects of curcumin against lead acetate-induced hepatotoxicity and nephrotoxicity in Wistar rats. Biological trace element research. 2015; 167(1):91-102.

\section{The Authors:}

Dr. Saba Amjad

Assistant Professor,

Department of Anatomy,

UCMD, University of Lahore

Prof. Muhammad Suhail

Head Department of Anatomy,

Federal Postgraduate Medical Institute, Lahore

Saima Malik

Associate Professor,

Department of Anatomy,

University Medical \& Dental College, Faisalabad
Tayyaba Muzaffar

Assistant Professor,

Department of Anatomy,

Federal Postgraduate Medical Institute, Lahore

Javaid Iqbal,

Associate Professor,

Department of Anatomy,

Federal Postgraduate Medical Institute, Lahore

Hadia Zulfiqar

Senior Demonstrator

Department of Anatomy,

Continental Medical College, Lahore

\section{Corresponding Author:}

Saba Amjad,

Assistant Professor,

Department of Anatomy,

UCMD, University of Lahore

E-mail:dr.saba14@hotmail.com 